The Astrophysical Journal, 459: L83-L86, 1996 March 10

(c) 1996. The American Astronomical Society. All rights reserved. Printed in U.S.A

\title{
PULSAR-WIND ORIGIN OF COSMIC-RAY POSITRONS
}

\author{
X. ChI, ${ }^{1}$ K. S. Cheng, ${ }^{2}$ And E. C. M. Young ${ }^{1}$ \\ Received 1995 April 10; accepted 1995 December 28
}

\begin{abstract}
We show that the electron-positron pairs produced in the magnetosphere of a pulsar can be accelerated monoenergetically to relativistic energies in the wind driven by low-frequency electromagnetic waves. The final achievable energy, depending on the surface magnetic field and rotation period of the individual pulsar, is mostly in the range $10^{-2}-10^{2} \mathrm{GeV}$. Based on the pair production rate of the individual pulsars and the Galactic pulsar population, the electron-positron flux from the Galactic pulsars is estimated from a statistical analysis of the observed pulsar data. We find that the flux is enough to account for the measured positron fraction and that its energy spectrum well matches the measured one. Our model predicts that the positron fraction will rise with increasing energy until its peak value at $30 \mathrm{GeV}$ and then drop off.
\end{abstract}

Subject headings: acceleration of particles — cosmic rays — pulsars: general

\section{INTRODUCTION}

The positrons in the primary cosmic-ray flux have been popularly considered to be secondary-the final decay products of pions created in the nuclear interactions of cosmic-ray protons with the interstellar gas (e.g., Ginzburg \& Syrovatski 1964; Giler, Wdowczyk, \& Wolfendale 1977; Webber 1980; Dogiel et al. 1987). Direct measurements (Fanselow et al. 1969; Buffington, Orth, \& Smoot 1975; Müller \& Tang 1987; Golden et al. 1994) of the positron content have covered a wide energy range, from 0.06 to $30 \mathrm{GeV}$. The data indicate that the positron fraction $e^{+} /\left(e^{-}+e^{+}\right)$varies significantly with energy, first decreasing from $40 \%$ to $5 \%$ with energy increasing from 0.06 to $2 \mathrm{GeV}$, then increasing rapidly again and reaching $20 \%$ at $17 \mathrm{GeV}$. Figure 1 presents a summary of the experimental data.

Many calculations of the fraction of secondary positrons have been done using various propagation models of cosmic rays, and the most extensive one is by Protheroe (1982). The results from all of the models predict a slowly decreasing trend with increasing energy and may be sufficient to account for the experimental data at $2 \mathrm{GeV}$. However, none of them can reproduce the measured rapid increase in the tens of $\mathrm{GeV}$ range. Further, if the measured high fraction at energies below $0.1 \mathrm{GeV}$ were regarded as secondary, the path length required for $\mathrm{GeV}$ cosmic-ray protons would be $1000 \mathrm{~g} \mathrm{~cm}^{-2}$ (Müller \& Tang 1990)! Thus, other origins should be sought for most of the Galactic cosmic-ray positrons. Previously, the potential positron sources have been suggested by ${ }^{56} \mathrm{Co} \beta$-decays in supernova remnants (Lingenfelter \& Ramaty 1979; Ellison, Frank, \& Ramaty 1990), pair productions by gamma-ray photons interacting with starlight photons in the interstellar medium (Mastichiadis, Protheroe, \& Stephen 1991), and gamma-ray cascade at the polar caps of pulsars (Harding \& Ramaty 1987; Boulares 1989). In this work, we present a pulsar-wind model for the origin of the bulk of the cosmic-ray positrons.

\footnotetext{
${ }^{1}$ Department of Physics and Materials Science, City University of Hong Kong, Tat Chee Avenue, Kowloon, HK.

2 Department of Physics, University of Hong Kong, Pokfulam Road, HK.
}

\section{PULSAR-WIND MODEL}

Pulsars are known by their periodic coherent radio emissions. It is generally believed that these coherent radio waves are emitted by extremely relativistic electrons accelerated inside an electrostatic acceleration region above the polar cap called the polar gap (Ruderman \& Sutherland 1975; Arons 1983; Rozental \& Usov 1985). Another inevitable product by the relativistic electrons is high-energy gamma rays. As these particles are restricted to move along the superstrong magnetic field $\left(\sim 10^{12} \mathrm{G}\right)$, they will radiate curvature photons with a characteristic energy (Ruderman \& Sutherland 1975)

$$
E_{\text {cur }}=\frac{3}{2} \gamma_{p}^{3} \frac{c}{S} \hbar=1.3 \times 10^{10} B_{12}^{-3 / 7} P^{-2 / 7} \mathrm{eV}
$$

where $\gamma_{p}$ is the Lorentz factor of the primary electrons, $s=\left(R r_{L}\right)^{1 / 2}$ is the curvature radius with the stellar radius $R=10^{6} \mathrm{~cm}$ and the light-cylinder distance $r_{L}=c / \Omega, B_{12}$ is the strength of the surface magnetic field in units of $10^{12} \mathrm{G}$, and $P$ is the rotation period in units of seconds.

Such curvature photons cannot escape from the pulsar since the magnetic field in the vicinity of the stellar surface is so strong that they will be converted into $e^{ \pm}$pairs by the local $B$ field (Erber 1966). It is these pairs (secondary pairs) that prevent the potential drop of the polar gap from growing unlimited. The typical potential drop of the polar gap at equilibrium is

$$
\Delta V=1.8 \times 10^{13} B_{12}^{-1 / 7} P^{1 / 7} \mathrm{~V} .
$$

Before escaping from the light cylinder, each of the primary electrons or positrons emits $N_{\mathrm{ph}}=e \Delta V / E_{\mathrm{cur}}$ curvature photons. There are

$$
\dot{N}=2.7 \times 10^{30} \mathrm{P}^{-2} B_{12} \text { pairs s}^{-1}
$$

primary electron-positrons passing through the polar gap (Goldreich \& Julian 1969). Thus, the rate of secondary $e^{ \pm}$ pairs emitted from the polar gap is

$$
\dot{N}_{ \pm}=3.7 \times 10^{33} B_{12}^{9 / 7} P^{-11 / 7} \text { pairs s }^{-1} .
$$

Most of these $e^{ \pm}$pairs are created beyond the polar gaps and are moving toward the light cylinder (Ruderman \& Sutherland 1975; Arons 1983), so they will eventually escape 


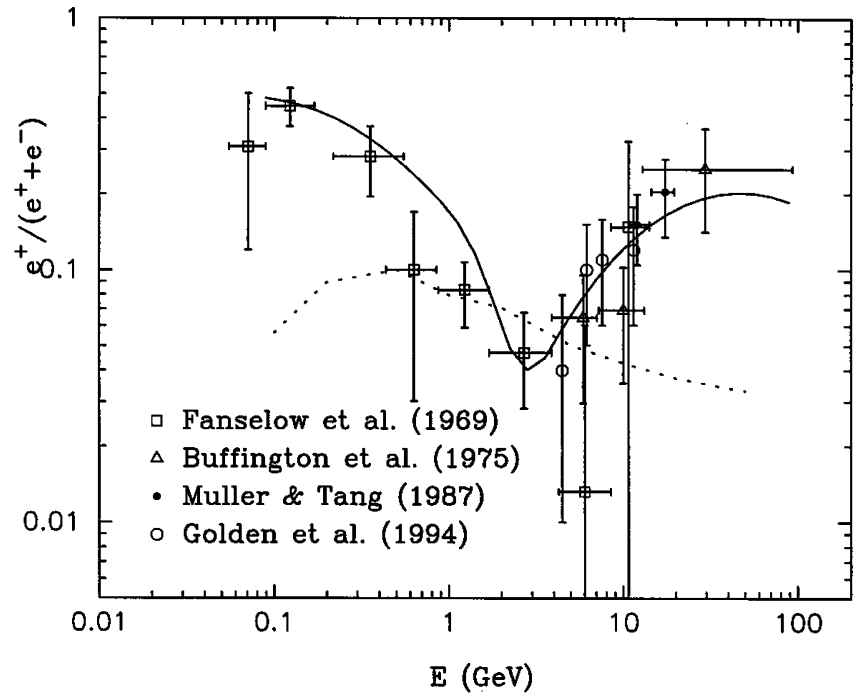

FIG. 1.-Positron fraction vs. energy in the primary cosmic-ray electron flux. The scattered points are a summary of the major experimental data. The dotted line represents the fraction of secondary positrons by Leaky Box Model (Protheroe 1982). The solid curve is the prediction of our pulsar-wind model.

from the light cylinder instead of being annihilated at the stellar surface. Beyond the light cylinder, they will incorporate the pulsar wind to flow out and be accelerated by the electromagnetic waves. It is well known that a pulsar loses its rotation energy via magnetic dipole radiation at frequency $P^{-1}$ Hz. The plasma frequency of the secondary $e^{ \pm}$pairs at distance $d$ from the pulsar is given by $f_{p}=(1 / 2 \pi)\left[\left(4 \pi n_{ \pm} e^{2}\right) /\right.$ $\left.m_{e}\right]^{1 / 2}$, with $n_{ \pm}=\dot{N}_{e^{ \pm}} /\left(4 \pi d^{2} c\right)$. We can see that $f_{p} \gg P^{-1}$ at the light cylinder. So the low-frequency electromagnetic wave will push the $e^{ \pm}$plasma until $f_{p}=P^{-1}$ or the electromagnetic wave and the plasma reach energy equipartition $n_{ \pm}(d) E_{e}=B^{2}(d) /$ $(4 \pi)$, where the $B$ field has the form $B=B_{s}\left(R / r_{L}\right)^{3}\left(r_{L} / d\right)$, in terms of the surface field $B_{s}$. From the above relation, we obtain the average energy of electrons in the plasma,

$$
E_{e}=8.1 \times 10^{9} B_{12}^{5 / 7} P^{-17 / 7} \mathrm{eV},
$$

for an individual pulsar. This energy estimate is consistent with the results of other theoretical work (Wilson \& Rees 1978; Kennel \& Coroniti 1984).

Apart from the polar cap, electron-positron pair production may occur more efficiently in the outer-magnetospheric region, the so-called outer gap (Cheng, Ho, \& Ruderman 1986), in the magnetosphere of some pulsars. A criterion for the existence of an outer gap is given by Cheng (1994). His argument is based on the fact that the pairs inside the gap can be produced via collisions between the thermal X-rays, with a typical energy $E_{\mathrm{x}}$ from the stellar surface heated up by the primary electron-positrons, and the curvature photons, with a typical energy $E_{\gamma}$ emitted by the primary electron-positrons inside the gap if $E_{\mathrm{x}} E_{\gamma} \geq\left(m_{e} c^{2}\right)^{2}$. This condition requires that the size of the outer gap $g$, being the ratio of the dimension of the outer gap to the light-cylinder radius, must satisfy the condition

$$
g=6.3 B_{12}^{-4 / 7} P^{9 / 7} \leq 1 .
$$

The pair production mechanism is a synchrotron photon cascade in a strong magnetic field. According to Halpern \&
Ruderman (1993), each primary electron-positron from an outer gap will still have an energy

$$
E_{p}=\gamma_{p} m c^{2}=5.7 \times 10^{12} P^{1 / 3} \mathrm{eV}
$$

before it strikes the neutron star surface. These primary electron-positrons will continue to emit curvature photons with a typical energy

$$
E_{\mathrm{cur}}=\frac{3}{2} \gamma_{p}^{3} \frac{c}{S} \hbar=6.4 \times 10^{8} P^{1 / 2} \mathrm{eV}
$$

These curvature photons will be converted into secondary $e^{ \pm}$ pairs in the local magnetic field when their energy satisfies (Ruderman \& Sutherland 1975)

$$
E_{\text {cur }} \geq \frac{2 m c^{2}}{15} \frac{B_{q}}{B}=3 B_{12} \mathrm{MeV} \equiv E_{\text {crit }},
$$

where $B_{q}=4.4 \times 10^{13} \mathrm{G}$ is a constant. The secondary $e^{ \pm}$pairs will lose their energies via the synchrotron radiation with photon energy

$$
E_{\text {syn }}=\frac{3}{2}\left(\frac{E_{\text {cur }}}{2 m c^{2}}\right)^{2} \frac{e \hbar B(r)}{m c}=2.6 \times 10^{8} B_{12} P\left(\frac{r}{3 R}\right)^{-3} \mathrm{eV} .
$$

We can see that normally $E_{\text {syn }}>E_{\text {crit }}$, so a photon-electron cascade will start and develop until this condition fails. At the end of a cascade, each incoming primary electron-positron can produce, on average,

$$
N_{e^{ \pm}}=\frac{E_{p}}{E_{\text {crit }}}=1.9 \times 10^{6} B_{12} P^{1 / 3} \text { pairs, }
$$

so the total pair production rate can be estimated,

$$
\dot{N}_{e^{ \pm}}=g \dot{N} N_{e^{ \pm}}=3.4 \times 10^{37} g B_{12}^{10 / 7} P^{-8 / 21} \mathrm{~s}^{-1},
$$

where $\dot{N}$ and $g$ are given by equations (3) and (6), respectively.

Since these pairs are created close to the stellar surface and the field lines are converging, only a small fraction may keep moving toward the star and annihilate on the stellar surface. Ho (1986) showed that the lost cone for these pairs will approach $\pi / 2$ (cf. eq. 29 of Ho 1986). In other words, most of the pairs will be reflected by magnetic mirroring effect and then move toward the light cylinder. Similar to the case of polar cap pair production, these particles will flow out with the pulsar wind and be accelerated by the low-frequency electromagnetic wave. The equipartition energy can be derived in a similar way:

$$
E_{e}=1.1 \times 10^{6} B_{12}^{4 / 7} P^{-76 / 21} \mathrm{eV} .
$$

In the pulsar wind, the low-frequency electromagnetic waves quickly transfer their energy to particles via magnetic field reconnection (Coroniti 1990) and thus the equipartition can quickly be achieved. After the equipartition, the particles are still coupled with the magnetic field on their way out, until the distance where the magnetic reconnection is completed and they can escape. Thus, adiabatic energy loss should be taken into account. The estimated escape distance (Coroniti 1990) is about 1 order of magnitude larger than the equipartition distance for the case of the wind blown by the Crab pulsar. Here we adopt this result for all pulsar winds and assume that the particle energy ejected into the interstellar medium is 1 
order of magnitude lower than the equipartition energy given by equations (5) and (13), respectively.

\section{THE GALACTIC POSITRON SPECTRUM}

Canonical pulsars are born in the explosion of supernovae. As the speed of pulsar proper motion is normally 1 or 2 orders of magnitude less than that of the initial supernova blast wave, the pulsar is immersed in the supernova remnant bubble for a certain period of time. During this period, the electronpositrons accelerated by pulsar winds will undergo expansion and thus their kinetic energy will fall due to adiabatic losses. Numerical simulations (Slavin \& Cox 1992) show that supernova expansions in the warm interstellar medium will slow down significantly after $10^{5} \mathrm{yr}$, so that the energetic electronpositrons may survive after this epoch. Here we adopt $10^{5}$ yr to be the starting time for pulsar wind production of energetic electron-positrons.

The electron-positron energy spectrum is calculated by adding up the contribution from each individual pulsar in the Galaxy. Each pulsar's contribution depends on its rotation period and surface magnetic field, as given by equations (4), (5), (12), and (13). Since we know only the relevant parameters of observed pulsars, those of unobserved pulsars are assumed to be the same as the observed ones if the age is the same. We divide the observed sample of pulsars (Taylor, Manchester, \& Lyne 1993) with ages greater than $10^{5} \mathrm{yr}$ into age bins. For each bin, we estimate the expected number of pulsars with a Galactic pulsar birthrate 0.01 per year and derive the number ratio of expected to observed. Then we multiply the contribution of each observed pulsar by the ratio according to its age in order to sum up the total Galactic spectrum, as shown in Figure 2. There are two parts in the spectrum; one is contributed by those pulsars with both outer gaps and polar gaps denoted by filled squares, and the other is by those with only polar gaps denoted by filled circles. The solid curves are smooth line fits, denoted by "PW." A significant dip at $2 \mathrm{GeV}$ in the spectrum results from the junction of the two parts. This feature appears to match the energy variation of the observed positron fraction.

\section{POSITRON FRACTION}

To calculate the positron fraction, the contribution of negatrons from supernova remnants (SNRs) should be taken into account and the Galactic ambient spectra should be derived. The first problem can be solved by estimating the energetics. A reasonable estimate of the total Galactic SNR output of electrons turns out to be $\sim 10^{40} \mathrm{~s}^{-1}$ above $1 \mathrm{GeV}$, with a power-law spectral index -2.2 . It is comparable to the total output from pulsar winds as plotted in Figure 2 (dotted line). The second problem is solved by substituting the ambient spectrum with the ejection one. The validity of this approximation can be justified by the fact that the propagation effect is minor for the question concerned with the calculation of the positron fraction. As summarized by Cesarsky (1980), the major propagation effect on the Galactic cosmic rays is energy-dependent diffusion that will steepen the spectrum by

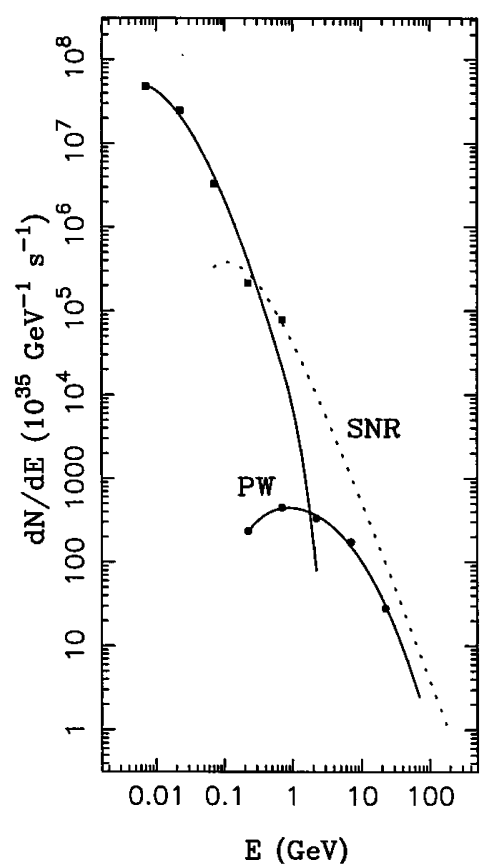

FIG. 2.-Total Galactic cosmic-ray electron spectra at ejection, in units of $10^{35} \mathrm{GeV}^{-1} \mathrm{~s}^{-1}$. The dotted line, labeled "SNR," represents the power-law spectrum with index -2.2 contributed by SNRs. The scatter points represent the electron-positron spectrum of the pulsar-wind model; the upper part originated from pulsar outer gaps and the lower part from pulsar polar gaps. The solid lines, labeled as "PW," are smooth curve fits to the theoretical points.

an index change of $0.3-0.6$ but will not make any systematic shift in the energy spectrum. Also, the Galactic electronpositrons in the energy range $1-30 \mathrm{GeV}$ will not suffer much energy loss due to inverse Compton scattering and synchrotron radiation. All the above effects are the same for positive and negative charged particles of the same magnetic rigidity. Energy-dependent effects of the solar modulation should not be significant at energies above $10 \mathrm{GeV}$.

To first-order approximation, the positron fraction is calculated directly from the ejection spectra shown in Figure 2. For the electron spectrum from SNRs, we make a cutoff at 0.1 $\mathrm{GeV}$ since it is quite uncertain below $1 \mathrm{GeV}$. The result is plotted in Figure 1 as the solid curve. Clearly, our model is able to reproduce the actual energy variation. It also predicts that the fraction will eventually reach its maximum at $30 \mathrm{GeV}$ and then go down at higher energies. In comparison, the polar cap cascade model (Harding \& Ramaty 1987; Boulares 1989) does not include the cooling process in the cap nor the acceleration in the wind. It predicts a slow increase of the positron fraction in the tens of GeV energy range, and the polar cap component will dominate over the SNR component at high energies in the electron spectrum.

We would like to thank A. W. Wolfendale for useful comments. This work is supported by a grant of the Hong Kong Research Grants Council.

\section{REFERENCES}

Arons, J. 1983, in Positron-Electron Pairs in Astrophysics, ed. M. Burns, A. K. Harding, \& R. Ramaty (New York: AIP), 163

Boulares, A. 1989, ApJ, 342, 807

Buffington, A., Orth, C. D., \& Smoot, G. F. 1975, ApJ, 199, 669
Cesarsky, C. J. 1980, ARA\&A, 18, 289

Cheng, K. S. 1994, in Proc. Towards a Major Atmospheric Cherenkov Detector III for TeV Astro-particle Physics, ed. T. Kifune (Tokyo: Tokyo Univ. Press), 
Cheng, K. S., Ho, C., \& Ruderman, M. 1986, ApJ, 300, 500

Coroniti, F. V. 1990, ApJ, 349, 538

Dogiel, V. A., Gurevich, A. V., Istomin, Ya. N., \& Zybin, K. P. 1987, MNRAS 228,843

Ellison, D. C., Frank, J. C., \& Ramaty, R. 1990, in Proc. 21st Int. Cosmic-Ray Conf., ed. R. J. Protheroe (Adelaide: Univ. Adelaide), 4

Erber, T. 1966, Rev. Mod. Phys., 38, 626

Fanselow, J. L., Hartman, R. C., Hildebrand, R. H., \& Meyer, P. 1969, ApJ, 158,771

Giler, M., Wdowczyk, J., \& Wolfendale, A. W. 1977, J. Phys. A., 10, 843

Ginzburg, V. L., \& Syrovatski, S. I. 1964, The Origin of Cosmic Rays (Oxford: Pergamon)

Golden, R. L., et al. 1994, ApJ, 436, 769

Goldreich, P., \& Julian, W. H. 1969, ApJ, 157, 869

Halpern, J. P., \& Ruderman, M. 1993, ApJ, 415, 286

Harding, A. K., \& Ramaty, R. 1987, in Proc. 20th Int. Cosmic-Ray Conf. (Moscow: Nauka), 2
Ho, C. 1986, MNRAS, 221, 523

Kennel, C. F., \& Coroniti, F. V. 1984, ApJ, 283, 710

Lingenfelter, R. E., \& Ramaty, R., 1979, in Proc. 16th Int. Cosmic-Ray Conf., ed. S. Miyake (Tokyo: Tokyo Univ. Press), 1

Mastichiadis, A., Protheroe, R. J., \& Stephen, S. A. 1991, in Proc. 22d Int Cosmic-Ray Conf., ed. D. O'Sullivan (Dublin: Dublin Institute), 2

Müller, D., \& Tang, K. K. 1987, ApJ, 312, 183

. 1990, in Proc. 21st Int. Cosmic-Ray Conf., ed. R. J. Protheroe (Adelaide: Univ. Adelaide), 3

Protheroe, R. J. 1982, ApJ, 254, 391

Rozental, I. L., \& Usov, V. V. 1985, Ap\&SS, 109, 365

Ruderman, M., \& Sutherland, P. 1975, ApJ, 199, 51

Slavin, J. D., \& Cox, D. P. 1992, ApJ, 392, 131

Taylor, J. H., Manchester, R. N., \& Lyne, A. G. 1993, ApJS, 88, 529

Webber, W. R. 1980, in Composition and Origin of Cosmic Rays, ed. M. M. Shapiro (Dordrecht: Reidel), 83

Wilson, D. B., \& Rees, M. J. 1978, MNRAS, 185, 297 